\title{
Retropharyngeal Calcific Tendonitis: Report of Two Cases
}

\author{
Rhea Victoria B. Razon, MD, Asad Nasir, MD, \\ George S. Wu, MD, Manal Soliman, MD, and Jeffrey Trilling, MD
}

Retropharyngeal calcific tendonitis is an inflammatory process of the superior oblique tendons of the longus colli muscle, a neck flexor in the upper cervical spine, caused by deposition of calcium hydroxyapatite crystals; the definitive diagnostic test is computed tomography (CT). Presented in this article are two cases seen at our institution. Patients typically present with acute onset of neck pain/spasm, odynophagia, dysphagia, and/or low grade fevers. Leukocytosis and elevated erythrocyte sedimentation rate may be noted. It is important to understand this entity because its signs and symptoms are mimickers of those of the more serious condition of retropharyngeal space abscess. Calcific tendonitis is managed conservatively whereas retropharyngeal abscess requires incision and drainage. Some may argue that this entity is a zebra because its reported incidence in the literature is low. However, most of these studies were done in an era when CT was not yet in vogue. With today's widespread use of CT and its superb ability to visualize the calcification, the true incidence of this condition is probably higher and, thus, it is important for the family practitioner to be aware of this entity. The astute clinician may save the patient from unnecessary diagnostic workup, undue anxiety, and delays in hospital discharge. ( $\mathrm{J}$ Am Board Fam Med 2009;22:84-88.)

\section{Case 1}

A 30-yr-old female with no significant past medical history presented with a 3-day history of rightsided neck pain, exacerbated by movement and intake of food. Two days prior, the patient was seen at a community hospital and diagnosed with torticollis. She was discharged with nonsteroidal antiinflammatory drugs (NSAIDs) and a muscle relaxant without significant improvement.

There was no history of strenuous activity, hoarseness, shortness of breath, nausea, vomiting, antecedent injury, neck pain, or arthritic disorder. White blood cell count was normal at 7900 cells per $\mathrm{mm}^{3}$. Blood culture showed no growth. Patient was afebrile.

This article was externally peer reviewed.

Submitted 8 February 2008; revised 14 March 2008; accepted 17 March 2008.

From the Departments of Family Medicine (RVBR, MS, JT), Internal Medicine (AN), and Radiology (GSW), Stony Brook University Hospital, New York.

Funding: none.

Conflict of interest: none declared.

Corresponding author: Rhea Victoria B. Razon, MD, Department of Family Medicine, Stony Brook Hospital, Nicholls Road, Level 4, Stony Brook, New York 11794 (Email: rheavictoria@yahoo.com).
The physical examination was positive for right para-cervical point tenderness and limited range of motion in all directions. There was no retropharyngeal asymmetry, bulge, or neck lymphadenopathy noted. The uvula was midline.

Cervical spine radiograph showed no fracture or subluxation; no abnormal calcification was noted. CT of the neck with intravenous contrast demonstrated fluid in the retropharyngeal space without wall enhancement, adjacent to the longus colli muscles. No definite abscess was noted. In retrospect, a small amorphous calcification was seen at the anterior C1-C2 level (see Figures $1-3)$. This crucial finding of calcification was missed by the radiologist; only the finding of the nonspecific fluid collection was noted in the radiology report. If the calcification had been noted, a definitive diagnosis of retropharyngeal calcific tendonitis could have been made, and the workup would have concluded. However, because the calcification was not picked up and the patient's symptoms were quite concerning, magnetic resonance imaging of the neck was performed, which again showed a retropharyngeal space fluid collection with extensive inflamma- 


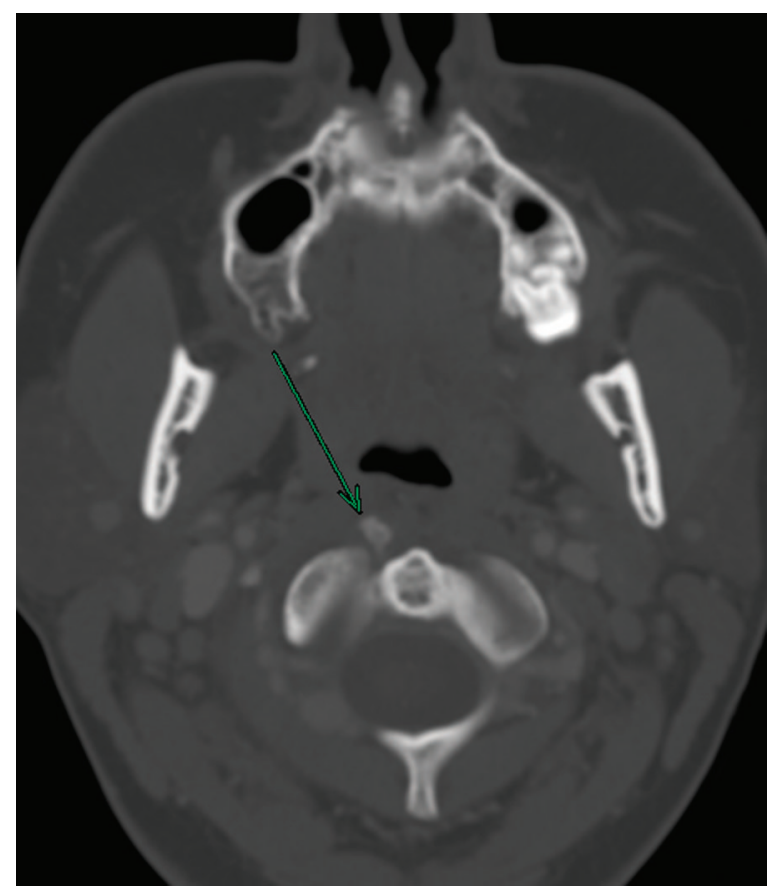

Figure 1. Axial neck computed tomography shows the amorphous calcification anterior to the C1-C2 level (arrow).

tory changes extending to the right carotid space (see Figure 4).

At admission, ear, nose, and throat and orthopedic surgery were consulted, and on reexamina-

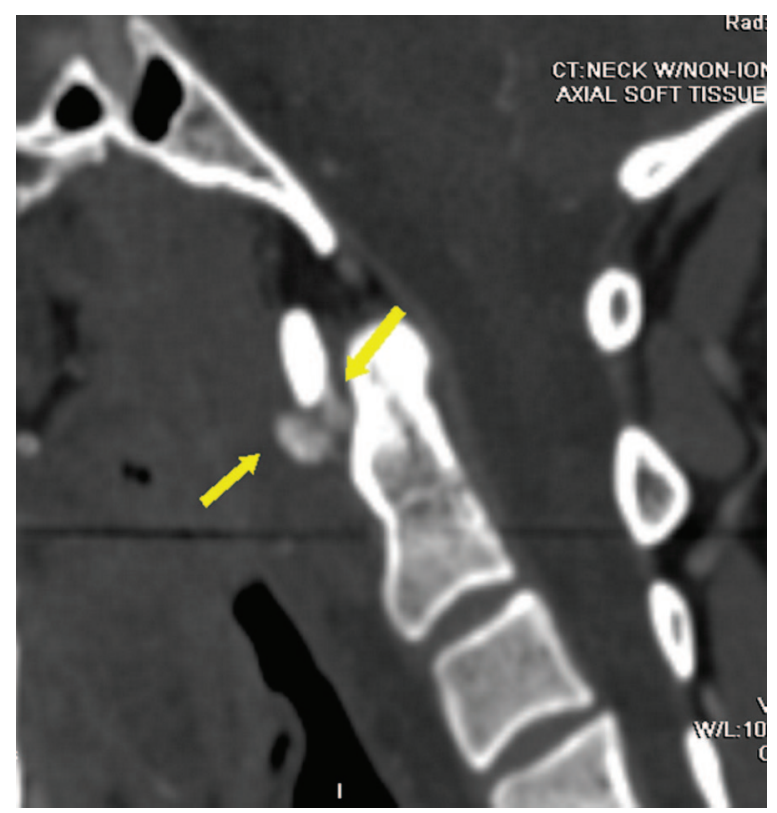

Figure 2. Sagittal neck computed tomography shows the same amorphous calcification at the C1-C2 level (arrow).

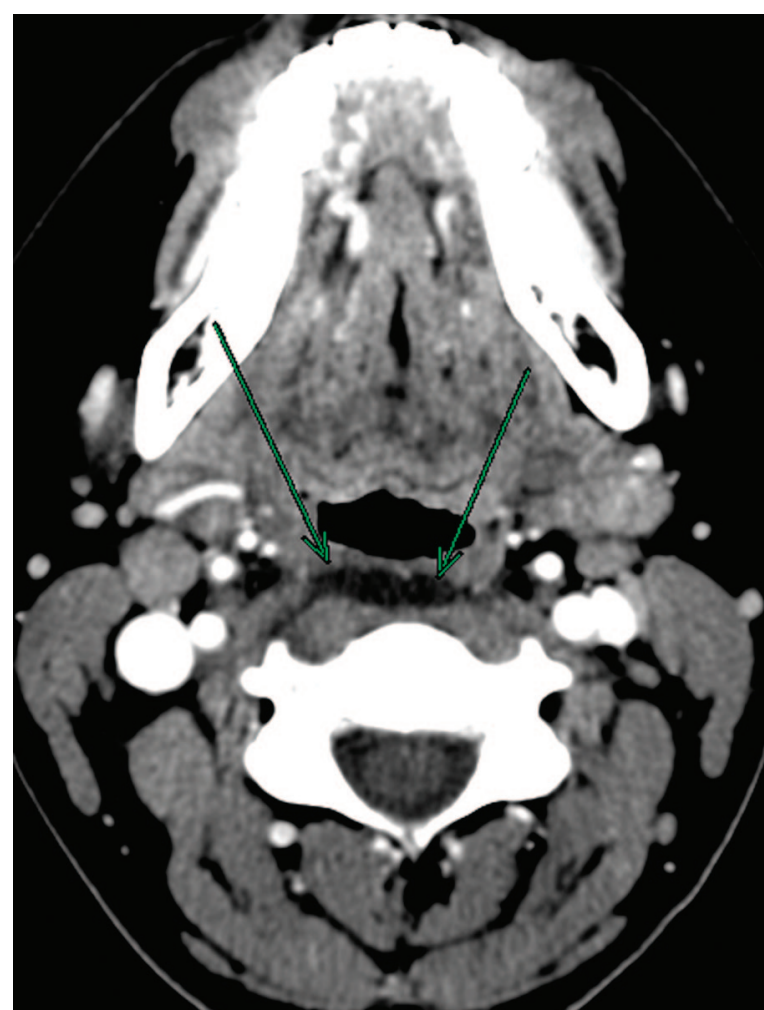

Figure 3. Axial neck computed tomography shows the dark retropharyngeal space fluid collection (arrow).

tion of the imaging studies, the calcification was now noted. Based on the characteristic image findings and the patient's clinical symptoms, patient was diagnosed with calcific retropharyngeal tendonitis and successfully treated with a course of NSAIDs for 14 days, analgesics, and tapered methylprednisolone for 5 days.

\section{Case 2}

A 43-yr-old male presented with a 6-day history of sore throat and posterior neck pain. He was initially seen by an outside provider and was treated with azithromycin and a muscle relaxant; however, his neck pain persisted with an interval onset of dysphagia. Outside neck CT was done. The attending radiologist picked up on the fluid collection anterior to the longus colli muscle but did not note the calcification (see Figures 5 and 6). Like the first patient, magnetic resonance imaging (MRI) was then done, which showed the previously noted retropharyngeal fluid collection (see Figure 7). His outside provider urged him to come to the emergency room for further evaluation. He was seen by ear, nose, and throat and was given the correct 


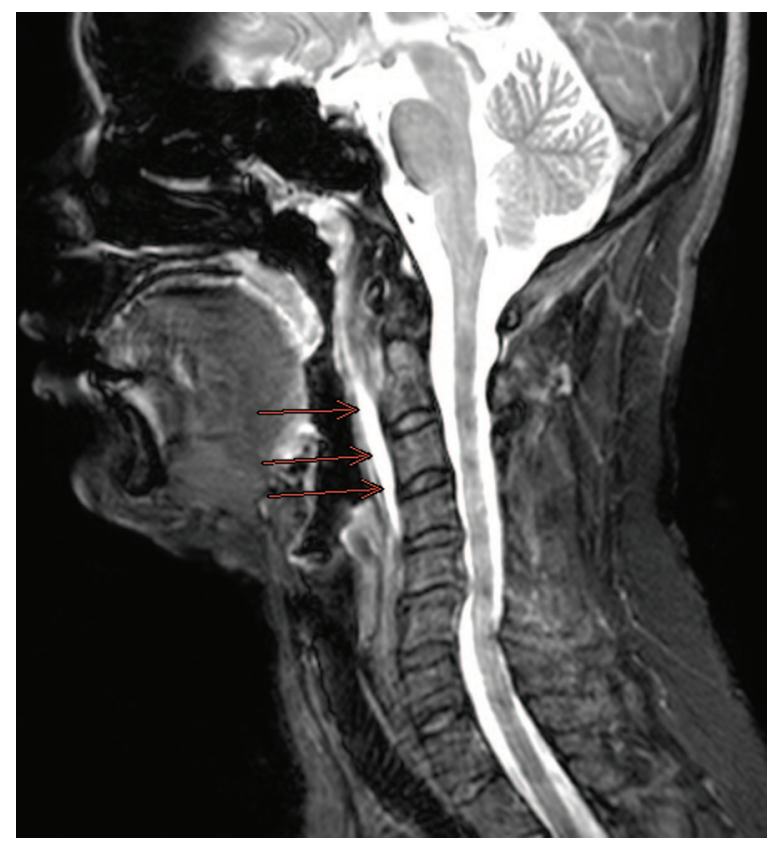

Figure 4. Sagittal T2 weighted neck magnetic resonance imaging shows the bright retropharyngeal space fluid collection from $\mathrm{C} 1$ to $\mathrm{C} 4$ (arrows). The calcification is very difficult to see on magnetic resonance imaging.

diagnosis of retropharyngeal calcific tendonitis. His admission laboratory values included slight leukocytosis of 10,900 cells per $\mathrm{mm}^{3}$ and a negative blood culture. The patient was afebrile.

The physical examination was positive for erythematous posterior pharynx, tenderness along the paraspinal areas bilaterally, small bilateral anterior

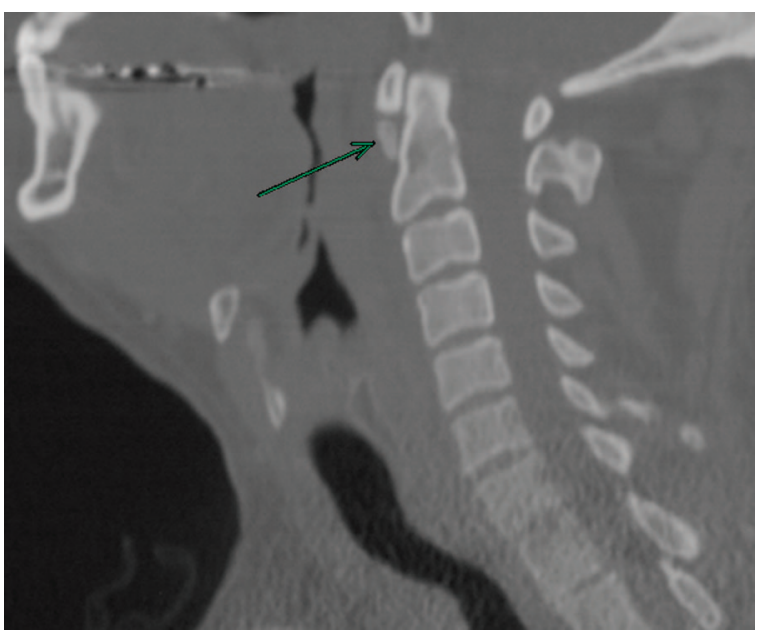

Figure 5. Axial neck computed tomography shows the amorphous calcification anterior to the C1-C2 level (arrow).

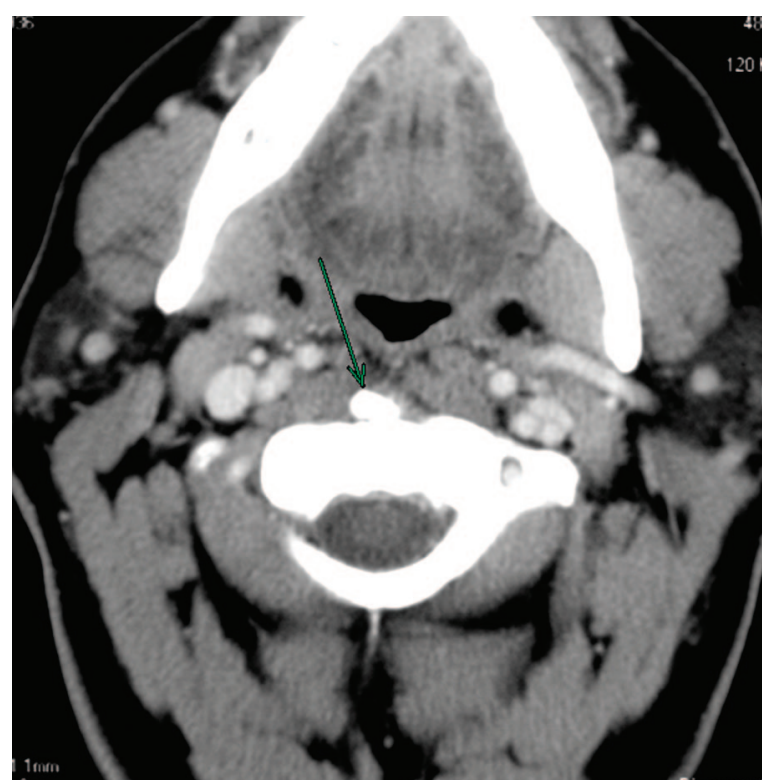

Figure 6. Sagittal neck computed tomography shows the same amorphous calcification (arrow).

cervical lymphadenopathy, and limited range of motion in all directions. There was no exudate or posterior pharyngeal bulge. The uvula was midline.

Review of outside CT of the neck showed nonenhancing retropharyngeal fluid collection and an amorphous calcification anterior to the longus colli muscle at the C1-C2 level.

His hospital treatment included 3 days of NSAIDs, analgesics, and 2 days of intravenous steroids. His home course treatment was 2 weeks of NSAIDs and tapered methylprednisolone with resolution of symptoms.

\section{Discussion}

Retropharyngeal calcific tendonitis, also known as acute calcific prevertebral tendonitis, is a clinical syndrome that was described originally by Hartley in $1964^{1,2}$ and was demonstrated by Ring and colleagues $^{3}$ in 1994 to be secondary to calcium hydroxyapatite deposition in the longus colli muscle. This muscle is a paired neck flexor muscle that comprises the prevertebral space. The longus colli muscle consists of 3 portions: superior oblique, inferior oblique, and vertical. Classically, the calcification affects the superior oblique portion of the longus colli muscle at the C1-C2 level. ${ }^{2}$ These superolateral fibers originate on the anterior tubercles of the transverse processes of the third to fifth 


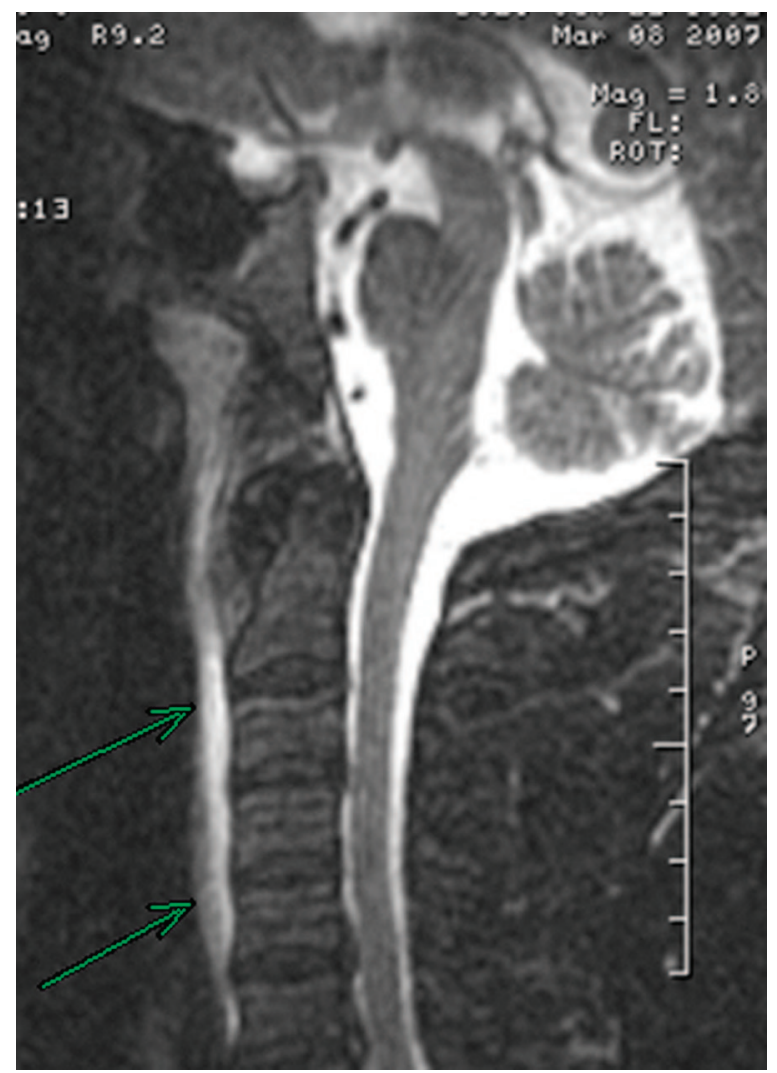

Figure 7. Sagittal neck magnetic resonance imaging shows the retropharyngeal space fluid collection (arrow).

cervical vertebrae and insert on the anterior tubercle of the atlas.

Proposed pathophysiology is that rupture of these calcific hydroxyapatite crystals provokes an inflammatory response in the surrounding longus colli muscle and leads to formation of reactive fluid in the retropharyngeal space surrounding the muscle. When the patient's symptoms resolve, the calcification also disappears. ${ }^{4}$

This entity may occur in association with underlying collagen vascular disorders, kidney failure, or osteoarthritis. ${ }^{5}$ The condition affects adults within a reported age range of 21 to 81 years, with greatest distribution between 30 and 60 years. The incidence of this condition is rare, although it is quite likely that the condition is underreported. ${ }^{2,6}$ Furthermore, with the recent prevalent use of CT, which is the best test for this condition, its true incidence is probably higher than previously thought.

Symptoms are secondary to inflammation of the longus colli and include acute to subacute onset of neck pain, dysphagia, odynophagia, and low-grade fever. The dysphagia is due to the close proximity of the retropharyngeal space to the adjacent pharyngeal constrictors.?

Examination typically reveals cervical paraspinal muscle spasm with the head held in slight flexion. The range of motion is extremely limited, usually secondary to severe pain, especially in extension. The white blood cell count and erythrocyte sedimentation rate may be mildly elevated, raising the suspicion of retropharyngeal infection.

Clinical findings of retropharyngeal calcific tendonitis strongly mimic those of a retropharyngeal space infection. It is difficult to separate lymphadenitis with abscess from calcific tendonitis based on history and lab findings, because the two overlap. Both disease entities may include fever, dysphagia, and elevated white blood cells and erythrocyte sedimentation rate. Imaging, specifically CT, is the key discriminant in separating the two disease entities. The importance of distinguishing between the two is that their clinical management differs considerably. In an abscess, antibiotics and incision and drainage are standard of care, whereas in tendonitis, conservative management with anti-inflammatories is the recommended course of care.

Lateral cervical radiograph may demonstrate calcifications anterior to the C1-C2 level. In a small percentage of patients, visible calcification is not detectable or is insufficient to allow the diagnosis to be made based on the lateral radiograph. ${ }^{2,6}$ In that case, the definitive diagnosis is by CT. CT scan shows the pathognomonic tendinous calcifications within the longus colli and can also demonstrate sterile fluid smoothly expanding the retropharyngeal space. ${ }^{7}$

In the presence of fluid collection, one must also consider the possibility of infection, specifically abscess from lymphadenitis. In lymphadenitis with suppuration, a fluid collection is seen, similar to that in calcific tendonitis; however, the fluid collection of infection typically has an enhancing rim on a CT scan with intravenous contrast. Adjacent necrotic lymph nodes with low attenuation centers may be seen. In addition, in lymphadenitis, calcification within the C1-C2 space, which is the hallmark of calcific tendonitis, is usually not seen.

The caveat, however, is that an abscess early in its development will not have enhancing walls. If the clinical suspicion of abscess is high, a repeat CT scan needs to be performed. If a fluid collection 
with an enhancing wall is subsequently demonstrated, then the diagnosis of abscess is very likely, even in the face of calcification within the longus colli muscles. MRI is not an optimal test because it is insensitive for the demonstration of calcification of the tendon. However, it may demonstrate high signal intensity on T2 weighted imaging corresponding to edema in the retropharyngeal space. ${ }^{6}$

Retropharyngeal calcific tendonitis, while generally a benign disorder, can potentially cause airway compromise if unrecognized and not treated. ${ }^{8}$ Treatment usually concentrates on pain alleviation using nonsteroidal anti-inflammatory therapy for 2 to 3 weeks, usually with resolution of symptoms within 72 hours. ${ }^{5}$ NSAIDs such as Indomethacin, Ibuprofen, or Naproxen are usually sufficient; however, some patients may require a short course of corticosteroids if the symptoms are severe. ${ }^{9}$ If symptoms persist for more than 2 weeks, another cause should be sought.

If calcific tendonitis is not treated, it is selflimiting and resolves within several weeks of onset of symptoms, although it is very painful and debilitating to the patient during that untreated time course. Familiarity with this rare entity and its pathognomonic imaging findings can prevent misdirected medical therapy and invasive attempts at surgical drainage. Furthermore, by being aware of this condition, one may save the patient from unnecessary diagnostic workup, undue anxiety, and delays in hospital discharge. If the report of a CT neck makes mention of a nonspecific fluid collec- tion in the retropharyngeal space, the astute clinician familiar with this condition may want to review the films with the radiologist to determine whether the $\mathrm{C} 1-\mathrm{C} 2$ calcification is present.

\section{References}

1. Hartley J. Acute cervical pain associated with retropharyngeal calcium deposit: a case report. J Bone Joint Surg 1964;46-A:1753-4.

2. Eastwood JD, Hudgins PA, Malone D. Retropharyngeal effusion in acute calcific prevertebral tendinitis: diagnosis with CT and MR imaging. AJNR Am J Neuroradiol 1998;19:1789-92.

3. Ring D, Vacarro AR, Scuderi G, Pathria MN, Garfin SR. Acute calcific retropharyngeal tendinitis. J Bone Joint Surg 1994;76-A:1636-42.

4. Hayes CW, Conway WF. Calcium hydroxyapatite deposition disease. Radiographics 1990;10:1031-48.

5. Smith RV, Rinaldi J, Hood DR, et al. Hydroxyapatite deposition disease: an uncommon cause of acute odynophagia. Otolaryngol Head Neck Surg 1996; 114:321-3.

6. Mihmanli I, Karaarslan E, Kanberoglu K. Inflammation of vertebral bone associated with acute calcific tendinitis of the longus colli muscle. Neuroradiology 2001;43:1098-101.

7. Omezzine SJ, Hafsa C, Lahmar I, Driss N, Hamza $\mathrm{H}$. Calcific tendinitis of the longus colli: diagnosis by CT. Joint Bone Spine 2008;75:90-1.

8. Guss DA, Jacoby I. Longus colli tendinitis causing acute neck pain. J Emerg Med 2002;22:211-2.

9. Kusunoki T, Muramoto D, Murata K. A case of calcific retropharyngeal tendinitis suspected to be a retropharyngeal abscess upon the first medical examination. Auris Nsus Larynx 2006;33:329-31. 